\title{
Review of: "Preliminary Findings of mRNA Covid-19 Vaccine Safety in Pregnant Persons"'"
}

\author{
Helene Banoun
}

Potential competing interests: The author(s) declared that no potential competing interests exist.

Preliminary Findings of mRNA Covid-19 Vaccine Safety in Pregnant Persons (April 21, 2021)

https://www.nejm.org/doi/full/10.1056/NEJMoa2104983

Authors' affiliations: none except that they all work at the CDC and furthermore:

Dr Liu and his spouse work for Ethicon|Johnson \& Johnson, particularly on surgical robotics, and own 50 shares of JNJ; the spouse also owns 20 shares of Moderna.

This is a post-marketing study that identifies real-life adverse effects of Covid vaccines in pregnant women

This is active smartphone monitoring of vaccinated pregnant women in the US (with Pfizer and Moderna mRNA vaccines).

The system sends sms messages with links to websites explaining AEs, and monitoring is carried out for 12 months.

During the first week: the system encourages reporting of AEs to VAERS, but only $14.7 \%$ of those identified are enrolled in the v-safe pregnancy register. These were almost exclusively health workers (94\%).

In the main study (December 14, 2020 to February 28, 2021) 72\% of the vaccinations took place during the first or second trimester of pregnancy and therefore these pregnancies were not completed at the time of publication.

Of the 3958 people included (out of a total of 35,691 pregnant women vaccinated, or $11 \%$ ) only 827 had a full-term pregnancy, or $21 \%$; the outcome for the remaining $79 \%$ is unknown.

From what we can understand, despite the lack of details provided, the pregnancies of women vaccinated during the third trimester between December and the end of February 2020 and who therefore gave birth between this period and April 2021 are of course counted.

The authors also include pregnancies terminated by miscarriage for women vaccinated in the first or second trimester, when these women reported the miscarriage to the surveillance system.

The number of women vaccinated in the first (before 14 weeks of pregnancy) and second trimester (between 14 and 28 weeks) in this study was 1224 and 1714 respectively, for a total of 2938.

96 miscarriages were reported before 13 weeks of gestation and 8 between 14 and 20 weeks, which gives $7.8 \%$ of miscarriages (with the approximation that miscarriages are reported before 13 weeks and pregnancies before 14).

Why did the authors not include this percentage in Table 4 on the line "miscarriage before 20 weeks"? Why did they not count the miscarriages before 20 weeks so that they could compare it to the usual rate (which, depending on the study, is 10 to $26 \%)$ ?

To have a valid comparison, it would have been necessary to count the women vaccinated before 20 weeks and to 
calculate the $\%$ of miscarriages before 20 weeks to be able to compare it to the usual rate. The authors of the study certainly have this data.

The 104/827 ratio is completely meaningless : 104 is the number of miscarriages before 20 weeks and 827 is the number of full-term pregnancies, the majority of which were in women vaccinated in the third trimester (after 28 weeks).

Proceeding in this way the authors obtain a percentage included in the usual range (12.6\% for a range between 10 and $26 \%$ ); whereas the percentage calculated according to their data before 13 weeks, of $7.8 \%$, was thus more favourable to the vaccine.

We also read, Table S4 (supplementary material, concerns AEs reported on VAERS only) that $50 \%$ of vaccinations took place during the first trimester of pregnancy, the most frequently reported SAE (serious adverse event) was miscarriage (46 in total, of which 37 in the first trimester).

Why did they not wait until all pregnancies were completed before publishing the study?

The conclusion of the article is worth reproducing

Limitations

"We were unable to evaluate adverse outcomes that might occur in association with exposures earlier in pregnancy, such as congenital anomalies, because no pregnant persons who were vaccinated early in pregnancy have had live births captured in the v-safe pregnancy registry to date; follow-up is ongoing. In addition, the proportion of pregnant persons who reported spontaneous abortion may not reflect true postvaccination proportions because participants might have been vaccinated after the period of greatest risk in the first trimester, and very early pregnancy losses might not be recognized. Whereas some pregnancies with vaccination in the first and early second trimester have been completed, the majority are ongoing, and a direct comparison of outcomes on the basis of timing of vaccination is needed to define the proportion of spontaneous abortions in this cohort. Because of sample-size constraints, both pregnancy and neo-natal outcomes were calculated as a proportion instead of a rate."

"Among pregnancy-specific conditions reported to the VAERS after Covid-19 vaccination, miscarriage was the most common. This is similar to what was observed during the influenza A (H1N1) pandemic in 2009 after the introduction of the $2009 \mathrm{H} 1 \mathrm{~N} 1$ inactivated influenza vaccine, where miscarriage was the most common adverse event reported by pregnant persons who received that vaccine. "

All of this leads one to believe that the rate of miscarriage after vaccination in the first trimester of pregnancy is higher than reported. 